\author{
Aline Silva-Costa ${ }^{\mathrm{a}}$ \\ (iD) https://orcid.org/0000-0003-1753-3922 \\ Bruna Pereira Braz ${ }^{\mathrm{b}}$ \\ (iD) https://orcid.org/0000-0002-4236-9151 \\ Rosane Härter Griep ${ }^{\text {b }}$ \\ (iD) https://orcid.org/0000-0002-6250-2036 \\ Lúcia Rotenberg ${ }^{\mathrm{b}}$ \\ (iD) https://orcid.org/0000-0002-4132-2167
}

a Universidade Federal do Triângulo Mineiro (UFTM), Departamento de Saúde Coletiva. Uberaba, MG, Brasil.

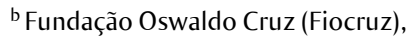
Instituto Oswaldo Cruz, Laboratório de Educação em Ambiente e Saúde. Rio de Janeiro, RJ, Brasil.

Contato:

Aline Silva-Costa

E-mail:

aline.costa@uftm.edu.br

Os autores declaram que o estudo recebeu apoio financeiro da Fundação de Amparo à Pesquisa do Estado do Rio de Janeiro - FAPERJ (E-26/111.5554/2008). Secretaria de Vigilância em Saúde do Ministério da Saúde - SVS/MS (182/2012). Griep RH e Rotenberg $L$ são bolsistas de Produtividade em Pesquisa do Conselho Nacional de Desenvolvimento Científico e Tecnológico - CNPq. Silva-Costa A foi bolsista de pós-doutorado em pesquisa do CNPq (150551/2015-0).

Os autores declaram não haver conflitos de interesses.

Os autores informam que este estudo é baseado na dissertação de mestrado de Bruna Pereira Braz, intitulada "O trabalho noturno e suas relações com a pressão arterial na equipe de enfermagem de um hospital público no Rio de Janeiro", defendido em 2017 na Escola Nacional de Saúde Pública Sergio Arouca, Fundação Oswaldo Cruz e que um resumo contendo parte deste estudo foi apresentado no Congresso Brasileiro de Epidemiologia em 2017.

\section{Trabalho noturno e pressão arterial: um estudo com foco nas doses de exposição}

\author{
Night shift work and blood pressure: focusing on exposure doses
}

\section{Resumo}

Objetivo: determinar se os níveis de exposição ao trabalho noturno (dose atual; dose acumulada) estão associados à hipertensão (HAS), pressão arterial sistólica (PAS) e pressão arterial diastólica (PAD). Métodos: estudo transversal realizado com 893 profissionais de enfermagem. Foram coletados dados sobre aspectos sociodemográficos, relacionados ao trabalho e a comportamentos de saúde. A pressão arterial foi aferida por meio de monitor digital. Resultados: após o ajuste pelas variáveis sociodemográficas, observou-se que trabalhar mais de 4 noites por quinzena foi associado ao aumento da PAS $(4,0 \mathrm{mmHg}$; intervalo de confiança [IC 95\%]: 1,01; 6,97) e PAD (2,3 mmHg; IC 95\%: 0,24; 4,35). O trabalho em mais de 4 noites por quinzena foi associado à ocorrência de hipertensão (RC 1,57; IC 95\%: $1,01 ; 2,43)$. Indivíduos que trabalharam à noite por mais de 9 anos apresentaram, em média, níveis de pressão arterial mais elevados (PAS de 3,7 mmHg [IC 95\%: 1,49; 5,92] e PAD de 2,0 mmHg [IC 95\%: 0,46; 3,52]), em comparação com aqueles que trabalharam à noite por 9 ou menos anos. Conclusão: esses resultados sugerem que os efeitos do trabalho noturno começam após uma certa dose de exposição, ou seja, após 9 anos de trabalho noturno ou quando exposto ao trabalho noturno por mais de 4 noites por quinzena.

Palavras-chaves: trabalho de turno; hipertensão; pressão sanguínea; enfermagem; saúde do trabalhador.

\begin{abstract}
Objective: to determine whether the levels of night work exposure (current dose; accumulated dose) are associated with hypertension (HBP), systolic blood pressure (SBP) and diastolic blood pressure (DBP). Methods: cross-sectional study of 893 nursing personnel. We collected data on sociodemographic, work-related and health behaviour factors and measured blood pressure using a digital monitor. Results: after adjusting for sociodemographic variables, working $>4$ nights per fortnight was associated with increased SBP (4.0mmHg; 95\% CI: 1.01; 6.97) and DBP (2.3 mmHg; 95\% CI: 0.24; 4.35). Working more than four nights per fortnight was associated to hypertension (OR 1.57; 95\% CI: 1.01; 2.43). Individuals who worked at night for $>9$ years displayed, on average, higher blood pressure levels (SBP of $3.7 \mathrm{mmHg}$ [95\% CI: 1.49; 5.92] and DBP of $2.0 \mathrm{mmHg}$ [95\% CI: 0.46; 3.52]), compared to those who worked at night for $\leq 9$ years. Conclusion: these findings suggest that effects of night work begin after a certain exposure dose, i.e, after 9 years of night work or when exposed to night work for more than 4 nights per fortnight.
\end{abstract}

Keywords: shift work; hypertension; blood pressure; nursing; occupational health. 


\section{Introdução}

Uma estimativa de 2017 indica que 7,5\% dos trabalhadores no Brasil eram trabalhadores noturnos, uma proporção maior entre os homens $(9,4 \%)$ do que entre as mulheres $(5,4 \%)^{1}$. Vários efeitos negativos à saúde são reconhecidos como relacionados a esse horário de trabalho ${ }^{2-5}$. O trabalho noturno é um fator de risco para hipertensão arterial (HA), e esta merece destaque não apenas porque sua prevalência é alta e crescente, mas também por ser um importante determinante de outras doenças cardiovasculares ${ }^{6}$.

Nas últimas décadas, diversos estudos avaliaram a associação entre trabalho noturno e pressão arterial ${ }^{4,7-8}$. Uma recente revisão sistemática com meta-análise mostrou associações estatisticamente significativas da HA com o trabalho em turnos rotativos, mas não com o trabalho noturno fixo ${ }^{4}$. Por outro lado, Ferguson et al. ${ }^{9}$ sugeriram que a exposição recente (menos de 12 meses) ao trabalho em turnos rotativos ou turnos noturnos fixos foi associada a maior incidência de hipertensão. $\mathrm{O}$ estudo destacou a maior incidência de HA entre os trabalhadores noturnos fixos, o que poderia ser explicado pelo desajuste circadiano, principalmente devido ao jet lag social vivenciado pelos trabalhadores noturnos ${ }^{9}$. Com base na porcentagem média mensal de turnos noturnos no ano anterior, foi observado que a baixa exposição ao trabalho noturno (0-5\%) estava associada a 2,3 vezes o risco de hipertensão enquanto trabalhar 95-100\% dos turnos noturnos aumentou o risco de hipertensão em 3,5 vezes em comparação com os trabalhadores não noturnos ${ }^{9}$.

Tais resultados colocam a necessidade de uma melhor compreensão dos efeitos do trabalho noturno - sua frequência e duração - à saúde. Nessa perspectiva, um artigo de revisão destacou a associação entre a exposição ao trabalho em turnos e o desenvolvimento de várias doenças, incluindo a hipertensão ${ }^{7}$. Porém, as horas de trabalho noturno não refletem necessariamente a frequência de exposição, que depende do número de noites trabalhadas dentro de um determinado período. Além disso, a probabilidade de casos de hipertensão é maior em trabalhadores diurnos com experiência anterior de trabalho noturno, em comparação com aqueles que nunca trabalharam à noite ${ }^{10}$.

Alguns autores apontam que resultados aparentemente divergentes podem decorrer de diferentes níveis de exposição ao trabalho noturno ${ }^{9-11}$, destacando a necessidade de estudos que avaliem como diferentes doses de exposição ao trabalho noturno afetam a HA.

Como o trabalho noturno é essencial para manter o atendimento ao paciente por 24 horas, é importante estudar a associação entre a dose de exposição ao trabalho noturno e a hipertensão, tanto para aprofundar a compreensão sobre tais relações quanto para colaborar com a definição das estratégias relacionadas à organização dos horários de trabalho nas instituições. Nesse sentido, esse estudo teve como objetivo verificar se diferentes níveis de exposição ao trabalho noturno (dose atual: número de noites trabalhadas; dose acumulada: anos de exposição ao trabalho noturno) estão associados à hipertensão (HA), pressão arterial sistólica (PAS) e pressão arterial diastólica (PAD) em trabalhadores de enfermagem.

\section{Métodos}

Nesse estudo transversal com profissionais de enfermagem de um hospital público do Rio de Janeiro, Brasil, todos aqueles que atuavam na assistência ao paciente foram convidados a participar $(\mathrm{N}=1332)$. Após recusas ( $\mathrm{n}=108 ; 8,1 \%)$, o estudo contou com 1224 trabalhadores. Desse total, 331 foram excluídos: 3 não conseguiram responder perguntas sobre trabalho noturno, 5 tomavam medicamentos anti-hipertensivos, 21 não tiveram a pressão arterial aferida (n = 21), 93 trabalharam à noite por menos de um ano e 209 relataram trabalhar em média menos de uma noite por semana. A amostra final foi composta por 893 trabalhadores noturnos (19h às 7h) ou ex-trabalhadores noturnos, ou seja, que trabalhavam das $7 \mathrm{~h}$ às $19 \mathrm{~h}$ no momento da coleta de dados, mas previamente expostos ao trabalho noturno.

Os dados foram coletados em 2013, no hospital, durante o expediente, usando um questionário adaptado de outros estudos ${ }^{10,12,13}$ para coletar informações sociodemográficas, profissionais e de saúde. As medidas antropométricas (peso e altura) e a pressão arterial dos participantes foram aferidas, como base em procedimentos padronizados, realizados por uma equipe treinada. Toda a coleta levou de 45 minutos a uma hora para cada trabalhador.

\section{Variáveis de exposição}

Dose atual de trabalho noturno: é o número de noites trabalhadas nas duas semanas anteriores. A dose atual foi calculada a partir da resposta à pergunta ${ }^{12}$ : Então, vamos lembrar em quais noites você trabalhou nas últimas duas semanas? A variável foi categorizada em: 2 a 4 noites por quinzena e mais de 4 noites por quinzena. Embora fossem trabalhadores noturnos fixos (turnos noturnos de 12 horas seguidos de folga de 60 horas), as trocas informais de dias de trabalho entre a equipe eram muito comuns, levando a noites consecutivas de trabalho.

Dose acumulada de trabalho noturno: tempo total de exposição ao trabalho noturno (em anos). A variável foi criada a partir das respostas às perguntas: Há quanto tempo você trabalha à noite? (para trabalhadores noturnos) e Por quanto tempo você trabalhou à noite? (para ex-trabalhadores noturnos). A variável foi categorizada em: 1 a 9 anos e mais de 9 anos de trabalho noturno ${ }^{12}$.

\section{Variáveis de resultado}

A pressão arterial foi aferida com um aparelho oscilométrico validado (Omron HEM 705CPINT). Um estudo que avaliou a precisão do Omron HEM-705-CP 
mostrou que o dispositivo pode ser usado para medir a pressão arterial em estudos de grande escala sem comprometer a validade ou a precisão do estudo ${ }^{14}$. A pressão arterial foi aferida em sala silenciosa, após 5 minutos de repouso, com o sujeito sentado. Três medições foram feitas em intervalos de um minuto. A média dessas medidas foi usada para determinar a PAS e a $\mathrm{PAD}^{14}$.

HA: medida pela pressão arterial (i) superior a $140 \mathrm{mmHg}$ para PAS ou (ii) superior a $90 \mathrm{mmHg}$ para PAD ou (iii) uso de medicamento anti-hipertensivo prescrito clinicamente.

\section{Covariáveis}

Foram obtidos dados sobre sexo, idade (menor ou igual a 40 anos; maior que 40 anos), raça/cor (branco; negro/pardo/amarelo), escolaridade (ensino médio; ensino superior), renda per capita (em salários mínimos nacionais [R $\$ 678,00$ em 2013]), estado civil (solteiro; casado; separado/divorciado; viúvo), categoria profissional (enfermeiro; técnico/auxiliar de enfermagem), atividade física (nenhuma; menor que 150 minutos/semana; maior ou igual a 150 minutos/ semana), hábitos de tabagismo (não fumante; ex-fumante; fumante), consumo de álcool (nunca, moderado; alto), queixa de insônia (sim, não), remédios anti-hipertensivos prescritos (sim; não) e índice de massa corporal (IMC em kg/m2, calculado pela divisão do peso dos participantes em quilogramas pelo quadrado de sua altura em metros). As medidas de peso foram realizadas em balança digital (Tanita ${ }^{\circledR}$, modelo Solar HS-301 que pode medir com precisão até $150 \mathrm{~kg}$ ) e a estatura em estadiômetro portátil (Alturexata ${ }^{\circledR}$ ), com escala de $0,1 \mathrm{~cm}$.

\section{Análise de dados}

Análises descritivas foram utilizadas para caracterizar a população do estudo. As variáveis categóricas foram apresentadas por frequência absoluta e relativa, e as variáveis quantitativas foram descritas a partir das medianas e intervalo interquartil (IIQ = P25-P75), médias e desvios-padrão (DP). Testes de qui-quadrado foram usados para comparação entre variáveis categóricas. O teste de Kolmogorov-Smirnov foi utilizado para verificar a suposição de normalidade. As variáveis quantitativas não apresentaram distribuição gaussiana, e o teste de Mann-Whitney foi escolhido para comparar dois grupos independentes.

Análises de regressão logística foram realizadas para testar a associação entre trabalho noturno e hipertensão. Razões de chances (RC) e intervalos de confiança de 95\% (IC 95\%) foram estimados.

Um modelo de regressão gama com função identidade foi usado para testar a associação entre trabalho noturno e PAS e PAD, uma vez que são desfechos contínuos e assimétricos. Foram estimados os coeficientes e intervalos de confiança de 95\% (IC 95\%).
Primeiramente, foram estimadas as associações brutas entre o trabalho noturno (dose atual e acumulada de trabalho noturno) e os desfechos (HA, PAS, PAD). Com base na literatura, os modelos foram ajustados por variáveis sociodemográficas: idade, sexo, raça/cor, escolaridade e categoria profissional. As variáveis de comportamento de saúde (atividade física, tabagismo, alcoolismo e queixas de insônia), potencialmente mediadoras da relação entre trabalho noturno e pressão arterial, não foram incluídas como ajustes no modelo de regressão ${ }^{8,9}$. Em uma análise de sensibilidade, o IMC foi avaliado como uma variável de confusão. A qualidade do ajuste foi avaliada usando o teste Hosmer-Lemeshow para regressão logística binária e o Critério de Informação de Akaike (AIC) e o teste Deviance para regressão gama. Adotou-se nível de significância estatística $\alpha=0,05$. Os dados foram analisados com o programa Statistical Package for the Social Sciences (SPSS®) versão 20.

Todos os trabalhadores responderam à seguinte pergunta adicional: "Algum médico já prescreveu algum medicamento para tratar a hipertensão?". Aqueles que responderam "Não, nunca” foram considerados em análises adicionais. Esse procedimento permitiu que a associação entre as doses de exposição ao trabalho noturno e a pressão arterial fosse testada em uma amostra mais homogênea do que na amostra total.

\section{Considerações éticas}

Esse estudo foi aprovado pelos comitês de ética em pesquisa do Instituto Oswaldo Cruz - IOC/ FIOCRUZ, no 635/11, aprovado em 12 de março de 2012. O estudo foi brevemente explicado aos participantes, que foram informados de que o envolvimento era voluntário e que poderiam se retirar a qualquer momento, sem implicações negativas. Cada participante recebeu um número de identificação e os nomes completos não foram registrados. Todos os participantes assinaram o termo de consentimento.

\section{Resultados}

Os participantes eram predominantemente do sexo feminino (84,0\%). A média de idade foi de $43,8(\mathrm{DP}=11,1)$ anos, a idade mínima de 24 anos e a máxima de 70 anos. A maioria dos participantes tinham ensino superior $(66,1 \%)$ e eram casados $(58,2 \%)$. A equipe de enfermagem foi dividida por ocupação, em auxiliar/técnico de enfermagem (64,6\%) e enfermeiros $(35,4 \%) ; 44,4 \%$ relataram ter dois ou mais empregos e $39,6 \%$ trabalharam mais de $40 \mathrm{~h}$ por semana. O tempo médio de trabalho noturno foi de 8 anos (54,4\% foram expostos ao trabalho noturno de 1 a 9 anos); a maioria (73,9\%) trabalhava de 2 a 4 noites por quinzena. A maioria (61\%) não praticava atividade física. A PAS média foi de 121,03 $\mathrm{mmHg}$ $(\mathrm{DP}=18,21 \mathrm{mmHg})$ e a PAD foi de $76,30 \mathrm{mmHg}$ $(\mathrm{DP}=11,16 \mathrm{mmHg})$. A prevalência de hipertensão 
foi de 38,2\%. Ao todo, 615 participantes nunca receberam prescrição de medicamentos para hipertensão.

Trabalhadores com maiores doses atuais de trabalho noturno (mais de 4 noites por quinzena) apresentaram maiores valores de PAS e PAD; este grupo também incluiu uma proporção maior de homens do que aqueles que trabalhavam até 4 noites por quinzena (Tabela 1).

Tabela 1 Dose atual de trabalho noturno por fatores sociodemográficos, ocupacionais e de saúde entre profissionais de enfermagem em um hospital público no Rio de Janeiro, Brasil, 2013 ( $\mathrm{N}=552$ trabalhadores noturnos atuais)

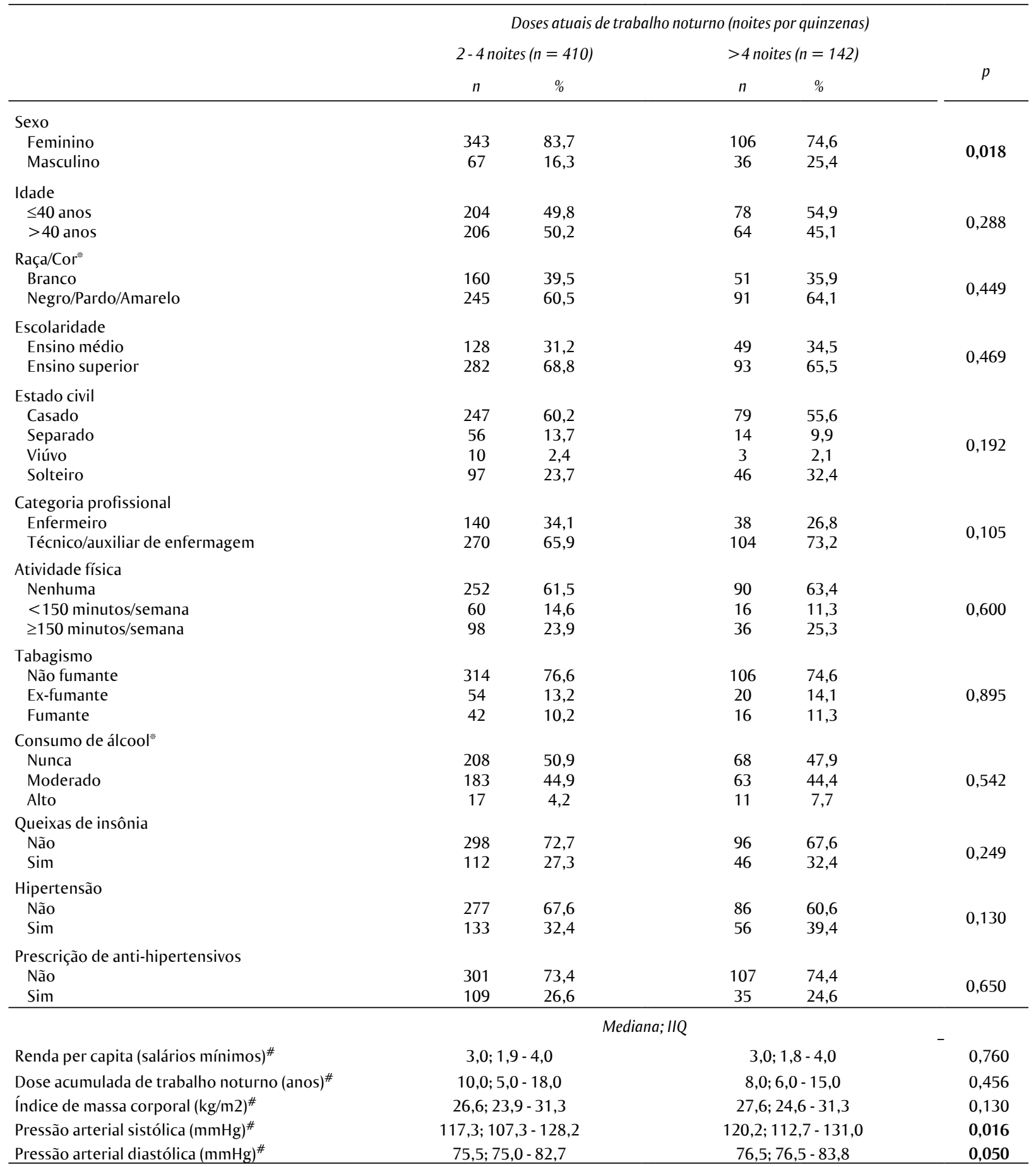

*Dados ausentes. Intervalo interquartil (IIQ) = P25-P75. Testes de qui-quadrado foram usados para comparação entre variáveis categóricas. ${ }^{\#}$ O teste de Mann-Whitney foi usado para comparar dois grupos independentes. 
Em comparação com indivíduos com uma baixa dose acumulada de trabalho noturno, aqueles com mais de 9 anos de trabalho noturno incluíram uma proporção maior de homens, idosos, indivíduos com escolaridade até o ensino médio, separados/divorciados/viúvos, fumantes ou ex-fumantes, hipertensos e pessoas com prescrição de anti-hipertensivos. Além disso, níveis mais elevados de IMC, PAS e PAD foram observados entre os trabalhadores com maiores doses acumuladas de exposição ao trabalho noturno do que entre os que trabalharam menos tempo de trabalho à noite (Tabela 2).

Tabela 2 Dose acumulada de trabalho noturno por fatores sociodemográficos, ocupacionais e de saúde entre profissionais de enfermagem em um hospital público no Rio de Janeiro, Brasil, 2013 ( $\mathrm{N}=893$ trabalhadores atualmente ou previamente expostos ao trabalho noturno)

\begin{tabular}{|c|c|c|c|c|c|}
\hline & \multicolumn{4}{|c|}{ Dose acumulada de trabalho noturno } & \multirow{3}{*}{$p$} \\
\hline & \multicolumn{2}{|c|}{$1-9 \operatorname{anos}(n=504)$} & \multicolumn{2}{|c|}{$>9 \operatorname{anos}(n=389)$} & \\
\hline & $n$ & $\%$ & $n$ & $\%$ & \\
\hline \multicolumn{6}{|l|}{ Sexo } \\
\hline Feminino & 445 & 88,3 & 305 & 78,4 & \multirow{2}{*}{$<0,001$} \\
\hline Masculino & 59 & 11,7 & 84 & 21,6 & \\
\hline \multicolumn{6}{|l|}{ Idade } \\
\hline$\leq 40$ anos & 306 & 60,7 & 92 & 23,7 & \multirow{2}{*}{$<0,001$} \\
\hline$>40$ anos & 198 & 39,3 & 297 & 76,3 & \\
\hline \multicolumn{6}{|l|}{ Raça/Cor** } \\
\hline Branco & 197 & 39,3 & 145 & 37,7 & \multirow{2}{*}{0,615} \\
\hline Negro/Pardo/Amarelo & 304 & 60,7 & 240 & 62,3 & \\
\hline \multicolumn{6}{|l|}{ Escolaridade } \\
\hline Ensino médio & 148 & 29,4 & 155 & 39,8 & \multirow[b]{2}{*}{0,001} \\
\hline Ensino superior & 356 & 70,6 & 234 & 60,2 & \\
\hline \multicolumn{6}{|l|}{ Estado civil } \\
\hline Casado & 304 & 60,3 & 216 & 55,5 & \multirow{4}{*}{0,020} \\
\hline Separado & 59 & 11,7 & 70 & 18,1 & \\
\hline Viúvo & 9 & 1,8 & 13 & 3,3 & \\
\hline Solteiro & 132 & 26,2 & 90 & 23,1 & \\
\hline \multicolumn{6}{|l|}{ Categoria profissional } \\
\hline Enfermeiro & 190 & 37,7 & 127 & 32,6 & \multirow{2}{*}{0,118} \\
\hline Técnico/auxiliar de enfermagem & 314 & 62,3 & 262 & 67,4 & \\
\hline \multicolumn{6}{|l|}{ Atividade física } \\
\hline Nenhuma & 293 & 58,1 & 252 & 64,8 & \multirow{3}{*}{0,059} \\
\hline$<150$ minutos/semana & 72 & 14,3 & 56 & 14,4 & \\
\hline$\geq 150$ minutos/semana & 139 & 27,6 & 81 & 20,8 & \\
\hline \multicolumn{6}{|l|}{ Tabagismo } \\
\hline Não fumante & 411 & 81,6 & 258 & 66,3 & \multirow{3}{*}{$<0,001$} \\
\hline Ex-fumante & 56 & 11,1 & 81 & 20,8 & \\
\hline Fumante & 37 & 7,3 & 50 & 12,9 & \\
\hline \multicolumn{6}{|l|}{ Consumo de álcool* } \\
\hline Nunca & 255 & 50,6 & 193 & 49,9 & \multirow{3}{*}{0,616} \\
\hline Moderado & 223 & 44,2 & 168 & 43,4 & \\
\hline Alto & 26 & 5,2 & 26 & 6,7 & \\
\hline Queixas de insônia* & & & & & \\
\hline Não & 357 & 70,8 & 268 & 69,1 & \\
\hline Sim & 147 & 29,2 & 120 & 30,9 & 0,569 \\
\hline Hipertensão & & & & & \\
\hline Não & 357 & 70,8 & 195 & 50,1 & \\
\hline Sim & 147 & 29,2 & 194 & 49,9 & $<0,001$ \\
\hline Prescrição de anti-hipertensivos & & & & & \\
\hline Não & 383 & 76,0 & 232 & 59,6 & $<0,001$ \\
\hline Sim & 112 & 24,0 & 157 & 40,4 & \\
\hline & & & & & \\
\hline Renda per capita (salários mínimos) & 3,0 & 4,0 & & 4,0 & 0,172 \\
\hline Índice de massa corp oral $\left(\mathrm{kg} / \mathrm{m}^{2}\right)^{\#}$ & 26,1 & 29,8 & 28,6 & $-32,4$ & $<0,001$ \\
\hline Pressão arterial sistólica $(\mathrm{mmHg})^{\#}$ & 114,7; & $-123,7$ & 124,0; & $-135,0$ & $<0,001$ \\
\hline Pressão arterial diastólica (mmHg) ${ }^{\#}$ & 73,7 & 80,7 & 77,7 & $-85,3$ & $<0,001$ \\
\hline
\end{tabular}

*Dados ausentes. Intervalo interquartil (IIQ) = P25-P75. Testes de qui-quadrado foram usados para comparação entre variáveis categóricas. \#O teste de Mann-Whitney foi usado para comparar dois grupos independentes. 
Trabalhadores classificados como hipertensos incluíam uma proporção maior de homens, indivíduos que eram mais velhos, pardos/negros, com escolaridade até o ensino médio, separados ou viúvos, técnicos de enfermagem e fumantes ou ex-fumantes. Ressalta-se ainda que 18,5\% dos indivíduos classificados como hipertensos pela aferição da pressão arterial relataram não ter recebido prescrição de anti-hipertensivos. Níveis mais elevados de IMC, PAS e PAD foram observados entre os hipertensos do que entre aqueles classificados como normotensos (Tabela 3).

Tabela 3 Hipertensão por fatores sociodemográficos, ocupacionais e de saúde entre profissionais de enfermagem em um hospital público no Rio de Janeiro, Brasil, 2013 ( $\mathrm{N}=893$ trabalhadores atualmente ou previamente expostos ao trabalho noturno)

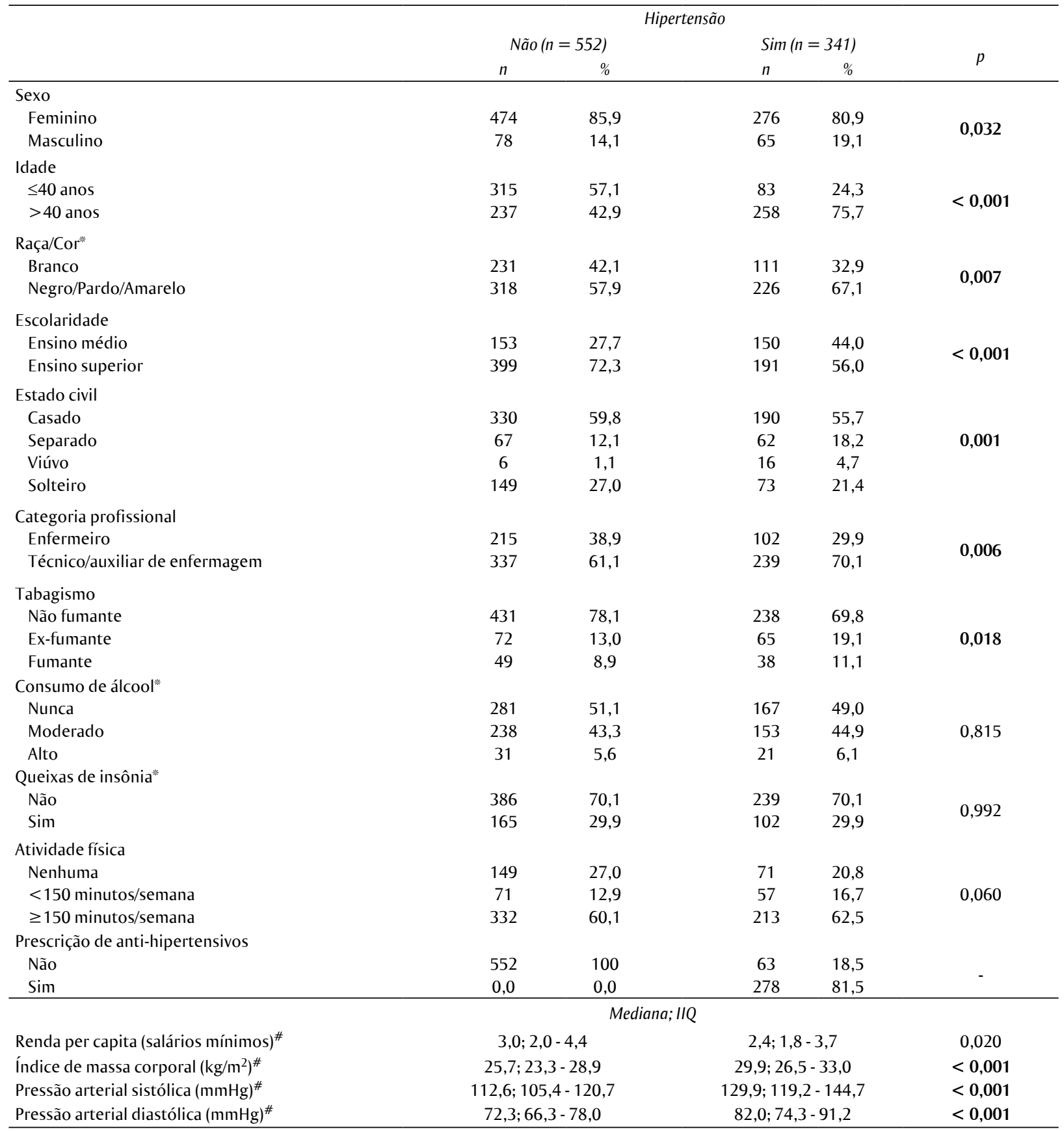

*Dados ausentes. Intervalo interquartil (IIQ) = P25-P75. Testes de qui-quadrado foram usados para comparação entre variáveis categóricas. ${ }^{\#}$ O teste de Mann-Whitney foi usado para comparar dois grupos independentes. 
Considerando os modelos de regressão ajustados para variáveis sociodemográficas, a maior dose atual de trabalho noturno (mais que 4 noites por quinzena) foi associada ao PAS e PAD aumentadas, em média, $4.0 \mathrm{mmHg}$ (IC 95\%: 1,01; 6,97 ) e $2.30 \mathrm{mmHg}$ (IC 95\%: 0,24; 4,35), respectivamente, em comparação ao grupo de referência. Além disso, o trabalho em mais de quatro noites por quinzena foi associado à hipertensão (RC 1,57 ; IC 95\%: 1,01; 2,43). Em relação à dose acumulada de trabalho noturno, aqueles que trabalharam à noite por mais de 9 anos apresentaram, em média, níveis de pressão arterial mais elevados (PAS de $3,7 \mathrm{mmHg}$ (IC 95\%: 1,49; 5,92) e PAD de 2,0 mmHg (IC 95\%: 0,$46 ; 3,52$ )) do que aqueles que trabalharam à noite por 9 ou menos anos. A associação entre hipertensão e dose acumulada de trabalho noturno foi limítrofe (Tabela 4).

Análises complementares ajustadas por variáveis sociodemográficas mais IMC retornaram resultados semelhantes. A maior dose atual de trabalho noturno (mais de 4 noites por quinzena) foi associada ao aumento da PAS e PAD, em média, de 3,6 mmHg (IC 95\%: 0,73; 6,41) e 1,94 $\mathrm{mmHg}$ (IC 95\%: 0,49; 3,84), respectivamente, em relação ao grupo de referência. Associação limítrofe foi observada entre a hipertensão e a dose atual de trabalho noturno $(\mathrm{RC}=1,51$; IC 95\%: 0,95; $2,41)$. Em relação à dose acumulada de trabalho noturno, indivíduos que trabalharam à noite por mais de 9 anos apresentaram, em média, níveis de PAS mais altos $(2,4 \mathrm{mmHg}$; IC 95\%: 0,22; 4,53). PAD $(0,88 \mathrm{mmHg}$; IC 95\%: -0,57; 2,33) e hipertensão (RC $=1,07$; IC 95\%: 0,76; 1,50) não mostraram associações estatisticamente significativas com a dose acumulada de trabalho noturno.

A Tabela 5 mostra os resultados adicionais para os indivíduos que nunca fizeram uso de anti-hipertensivos. Nesse grupo, os modelos de regressão ajustados mostraram que a maior dose acumulada de trabalho noturno foi associada com a PAS e PAD aumentada, em média, de $4,0 \mathrm{mmHg}$ (IC 95\%: 1,62; 6,38) e 2,1 mmHg, respectivamente. Também houve associação estatisticamente significativa entre maior dose acumulada de trabalho noturno e hipertensão (RC 1,88; IC95\% 1,04; 3,42). Aqueles que trabalharam mais noites apresentaram maior PAS $(4,7 \mathrm{mmHg}$; IC 95\%: 1,$73 ; 7,68)$ e PAD (2,9 mmHg; IC 95\%: 0,61; 5,13) do que o grupo que trabalhou menos noites por quinzena. A maior dose acumulada de trabalho noturno também foi fortemente associada à hipertensão (RC 3,06; IC95\% 1,54; 6,05).

Tabela 4 Associação entre doses de exposição ao trabalho noturno e pressão arterial entre profissionais de enfermagem em um hospital público no Rio de Janeiro, Brasil, 2013

\begin{tabular}{|c|c|c|c|c|c|c|}
\hline & \multicolumn{2}{|c|}{ Pressão arterial sistólica } & \multicolumn{2}{|c|}{ Pressão arterial diastólica } & \multicolumn{2}{|c|}{ Hipertensão } \\
\hline & Modelo bruto & $\begin{array}{l}\text { Modelo } \\
\text { ajustado }\end{array}$ & Modelo bruto & $\begin{array}{c}\text { Modelo } \\
\text { ajustado }\end{array}$ & Modelo bruto & $\begin{array}{c}\text { Modelo } \\
\text { ajustado }\end{array}$ \\
\hline & $\begin{array}{c}\beta \\
\text { (IC 95\%) }\end{array}$ & $\begin{array}{c}\beta \\
\text { (IC 95\%) }\end{array}$ & $\begin{array}{c}\beta \\
\text { (IC 95\%) }\end{array}$ & $\begin{array}{c}\beta \\
\text { (IC 95\%) }\end{array}$ & $\begin{array}{c}R C \\
(I C 95 \%)\end{array}$ & $\begin{array}{c}R C \\
(I C 95 \%)\end{array}$ \\
\hline \multicolumn{7}{|c|}{ Dose atual de trabalho noturno $(N=552)$} \\
\hline 2 - 4 noites/quinzena & Ref. & Ref. & Ref. & Ref. & Ref. & Ref. \\
\hline$>4$ noites/quinzena & $\begin{array}{c}4,1 \\
(0,69 ; 7,43)^{*}\end{array}$ & $\begin{array}{c}4,0 \\
(1,01 ; 6,97)^{*}\end{array}$ & $\begin{array}{c}2,3 \\
(0,14 ; 4,41)^{*}\end{array}$ & $\begin{array}{c}2,3 \\
(0,24 ; 4,35)^{*}\end{array}$ & $\begin{array}{c}1,37 \\
(0,93 ; 2,01)\end{array}$ & $\begin{array}{c}1,57 \\
(1,01 ; 2,43)^{*}\end{array}$ \\
\hline \multicolumn{7}{|c|}{ Dose acumulada de trabalho noturno $(N=893)$} \\
\hline $1-9$ anos & Ref. & Ref. & Ref. & Ref. & Ref. & Ref. \\
\hline$>9$ anos & $\begin{array}{c}9,5 \\
(7,22 ; 11,70)^{*}\end{array}$ & $\begin{array}{c}3,7 \\
(1,49 ; 5,92)^{*}\end{array}$ & $\begin{array}{c}4,0 \\
(2,52 ; 5,41)^{*}\end{array}$ & $\begin{array}{c}2,0 \\
(0,46 ; 3,52)^{*}\end{array}$ & $\begin{array}{c}2,39 \\
(1,81 ; 3,16)^{*}\end{array}$ & $\begin{array}{c}1,33 \\
(0,97 ; 1,82)\end{array}$ \\
\hline
\end{tabular}

Modelos ajustados para sexo, idade, raça/etnia, escolaridade e categoria profissional. Valor $\beta$ : diferença entre grupos, coeficientes derivados dos modelos de regressão gama. RC: Razão de Chances a partir de modelos de regressão logística. IC 95\%: intervalo de confiança de 95\%; Ref.: Categoria de referência. *Associação estatisticamente significativa. 
Tabela 5 Associação entre doses de exposição ao trabalho noturno e pressão arterial entre profissionais de enfermagem sem prescrição de anti-hipertensivos em um hospital público do Rio de Janeiro, Brasil, 2013

\begin{tabular}{|c|c|c|c|c|c|c|}
\hline & \multicolumn{2}{|c|}{ Pressão arterial sistólica } & \multicolumn{2}{|c|}{ Pressão arterial diastólica } & \multicolumn{2}{|c|}{ Hipertensão } \\
\hline & Modelo bruto & $\begin{array}{l}\text { Modelo } \\
\text { ajustado }\end{array}$ & Modelo bruto & $\begin{array}{l}\text { Modelo } \\
\text { ajustado }\end{array}$ & $\begin{array}{c}\text { Modelo } \\
\text { bruto }\end{array}$ & Modelo ajustado \\
\hline & $\begin{array}{c}\beta \\
\text { (IC 95\%) }\end{array}$ & $\begin{array}{c}\beta \\
(I C 95 \%)\end{array}$ & $\begin{array}{c}\beta \\
\text { (IC 95\%) }\end{array}$ & $\begin{array}{c}\beta \\
\text { (IC 95\%) }\end{array}$ & $\begin{array}{c}R C \\
\text { (IC 95\%) }\end{array}$ & $\begin{array}{c}R C \\
(I C 95 \%)\end{array}$ \\
\hline \multicolumn{7}{|c|}{ Dose atual de trabalho noturno $(n=408)$} \\
\hline 2 - 4 noites/quinzena & Ref. & Ref. & Ref. & Ref. & Ref. & Ref. \\
\hline$>4$ noites/quinzena & $\begin{array}{c}4,8 \\
(1,47 ; 8,09)^{*}\end{array}$ & $\begin{array}{c}4,7 \\
(1,73 ; 7,68)^{*}\end{array}$ & $\begin{array}{c}2,8 \\
(0,52 ; 5,16)^{*}\end{array}$ & $\begin{array}{c}2,9 \\
(0,61 ; 5,13)^{*}\end{array}$ & $\begin{array}{c}2,80 \\
(1,48 ; 5,27)^{*}\end{array}$ & $\begin{array}{c}3,06 \\
(1,54 ; 6,05)^{*}\end{array}$ \\
\hline \multicolumn{7}{|c|}{ Dose acumulada de trabalho noturno $(n=615)$} \\
\hline $1-9$ anos & Ref. & Ref. & Ref. & Ref. & Ref. & Ref. \\
\hline$>9$ anos & $\begin{array}{c}7,8 \\
(5,48 ; 10,18)^{*}\end{array}$ & $\begin{array}{c}4,0 \\
(1,62 ; 6,38)^{*}\end{array}$ & $\begin{array}{c}3,8 \\
(2,14 ; 5,52)^{*}\end{array}$ & $\begin{array}{c}2,1 \\
(0,32 ; 3,94)^{*}\end{array}$ & $\begin{array}{c}2,60 \\
(1,53 ; 4,43)^{*}\end{array}$ & $\begin{array}{c}1,88 \\
(1,04 ; 3,42)^{*}\end{array}$ \\
\hline
\end{tabular}

Modelos ajustados para sexo, idade, raça/etnia, escolaridade e categoria profissional. Valor $\beta$ : Diferença entre grupos, coeficientes derivados dos modelos de regressão gama. RC: Razão de Chances a partir de modelos de regressão logística. IC 95\%: intervalo de confiança de 95\%. Ref.: Categoria de referência. *Associação estatisticamente significativa.

\section{Discussão}

As associações estatisticamente significativas encontradas entre maior dose acumulada de trabalho noturno e níveis mais elevados de pressão arterial (PAS e PAD) e maiores chances de hipertensão, em comparação com a menor dose acumulada de trabalho noturno, são consistentes com estudos que mostraram associações significativas entre durações mais longas de trabalho noturno e níveis mais elevados de pressão arterial e maior probabilidade de hipertensão ${ }^{15,16}$.

Wang et al. ${ }^{15}$, que compararam características de mulheres que trabalhavam à noite e durante o dia, observaram que quanto maior a duração do trabalho noturno (em anos), maior a probabilidade de hipertensão, obesidade e tabagismo. Foram encontradas associações significativas com esses fatores de risco cardiovascular, principalmente em trabalhadoras com longa exposição ao trabalho noturno (mais de 20 anos), em comparação com aquelas que trabalhavam durante o dia ${ }^{15}$. Em um estudo de coorte prospectivo com enfermeiros, a maior duração do trabalho noturno rotativo foi associada a um maior risco de doença coronariana ${ }^{17}$. Esses resultados corroboram nossa hipótese de que maiores doses acumuladas de trabalho noturno estão associadas a níveis mais elevados de pressão arterial e maiores chances de hipertensão. Por outro lado, uma meta-análise mostrou associação significativa entre trabalho em turnos rotativos (mas não entre trabalhadores noturnos fixos) e hipertensão. No entanto, não explorou os efeitos das doses de exposição no trabalho noturno ${ }^{4}$.

Em relação ao detalhamento da exposição ao trabalho noturno por meio da investigação da atual dose de trabalho, nossos dados sugerem que, entre profissionais de enfermagem, maior frequência de trabalho noturno está associada a níveis elevados de pressão arterial e maiores chances de hipertensão. Um estudo de coorte recente mostrou que o trabalho noturno e em turnos rotativos estava associado a um maior risco de hipertensão. Em consonância com nossos resultados, aquele estudo constatou que, no período de um ano, quanto maior a frequência de trabalho noturno, maior o risco de hipertensão ${ }^{9}$. Estudos com outros desfechos mostraram resultados semelhantes. Peplonska et al. ${ }^{18}$ utilizaram várias medidas para avaliar a exposição ao trabalho noturno, incluindo horas de trabalho, noites trabalhadas e anos acumulados de trabalho noturno, ao estudar sua associação com a obesidade em enfermeiras e parteiras. Observaram associação entre maior frequência de trabalho noturno (mais de 8 noites por mês) e maiores chances de obesidade, em comparação com menor frequência de trabalho noturno (2-7 noites por mês). Mulheres que trabalharam mais à noite (turno noturno fixo) apresentaram maior risco de obesidade do que mulheres que trabalharam em turnos rotativos ${ }^{19}$.

Park et al. ${ }^{20}$ usaram o acúmulo de trabalho noturno, calculado pela multiplicação das noites por meses de trabalho, para mostrar que o trabalho noturno cumulativo foi significativamente associado a alto risco de doenças cardiovasculares, em comparação com quem nunca trabalhou à noite ${ }^{20}$. Tal contribuição metodológica reforça a importância da utilização da dose de trabalho noturno para avaliar o desfecho de interesse. Assim como nos estudos acima, os autores do presente estudo buscaram 
refinar a variável do trabalho por tempo de exposição e frequência do trabalho noturno (ou seja, dose acumulada e dose atual, respectivamente), levando a maior contribuição para a área do conhecimento e fortalecendo o estudo.

As doses de trabalho noturno consideradas nesse estudo apresentaram resultados convergentes em relação aos desfechos aqui avaliados (PAS, PAD e HA). No entanto, as variáveis de exposição são conceitualmente diferentes e referem-se a diferentes características de trabalho. A dose atual está relacionada às características do trabalho e suas consequências agudas, enquanto a dose acumulada reflete a vida do trabalhador ao longo dos anos, para considerar os efeitos crônicos de tal horário de trabalho. A duração do trabalho noturno não explica a frequência do trabalho noturno, que pode ser substancialmente diferente entre os sujeitos dependendo do número de noites trabalhadas ${ }^{18}$. No presente estudo, a dose acumulada de trabalho noturno também considera a experiência prévia no trabalho noturno por aqueles que atualmente trabalham durante o dia. Estudos anteriores do nosso grupo mostraram os efeitos do trabalho noturno prévio (entre os trabalhadores diurnos), em comparação com aqueles que nunca trabalharam à noite ${ }^{10,12,21}$.

Os impactos da exposição ao trabalho noturno não foram totalmente descritos. Os possíveis mecanismos pelos quais o trabalho noturno pode aumentar o risco de doenças cardiovasculares (incluindo hipertensão) decorrem da dessincronização circadiana relacionada a mudanças comportamentais, psicossociais e biológicas ${ }^{22}$. É preciso considerar que os ritmos circadianos governam as variáveis biológicas, entre elas a pressão arterial ${ }^{23}$. A relação entre trabalho noturno e hipertensão pode envolver mecanismos biológicos que influenciam os padrões de sono dos trabalhadores, induzindo atividade simpática mais intensa durante o sono $^{24}$, ritmo alterado da pressão sanguínea, com consequente ausência de sono $^{25}$, redução da regulação autonômica cardíaca ${ }^{8}$, menor duração do sono e aumento da sonolência ${ }^{26}$. Processos inflamatórios ${ }^{27}$ e alteração na regulação hormonal ${ }^{28}$ também podem estar envolvidos. Além disso, hábitos de comportamento não saudáveis são considerados mediadores no desenvolvimento da hipertensão em trabalhadores noturnos; entre os trabalhadores noturnos são observadas maiores frequências de consumo de alimentos com alto teor de gordura, refeições e lanches irregulares ${ }^{29}$, pouco envolvimento em atividades de lazer ${ }^{30}$ e maior prevalência de fumantes ${ }^{31}$. Todos esses fatores estão interligados devido à dessincronização circadiana e são considerados condições estressantes para a saúde cardiovascular ${ }^{22}$. Embora o presente estudo não tenha se concentrado em avaliar a relação da dose de trabalho noturno e da pressão arterial, independentemente do papel das variáveis mediadoras, é interessante notar que mesmo com ajuste adicional para o IMC, as associações entre a dose atual de trabalho noturno e o aumento dos níveis da pressão arterial continuaram estatisticamente significativas, embora com magnitudes menores, como esperado.

Tendo em vista as conhecidas alterações do ritmo circadiano decorrentes do trabalho noturno ${ }^{22,28}$, é oportuno considerar os possíveis efeitos dos medicamentos hipertensivos em trabalhadores noturnos. $\mathrm{O}$ trabalho noturno pode interferir nos padrões de dose-resposta de muitos medicamentos. Hermida et al. ${ }^{23}$ investigaram o efeito da administração de medicamentos para hipertensão respeitando a variação circadiana da pressão arterial entre hipertensos. Os autores observaram que o uso de drogas à noite era mais eficiente no controle da pressão arterial, diminuindo a prevalência de ausência de descenso noturno da pressão arterial e reduzindo a morbimortalidade por doenças cardiovasculares, do que o uso no horário tradicional, ou seja, ao acordar ${ }^{23}$. Assim, dados os efeitos que o trabalho noturno pode causar no ritmo circadiano da pressão arterial, com consequentes alterações na resposta aos medicamentos hipertensivos, a análise aqui realizada, considerando a possível interferência da prescrição de medicamentos, também apresentou resultados relevantes.

Ressalta-se, a esse respeito, que na subamostra em que nunca foram prescritos anti-hipertensivos, uma proporção não desprezível de indivíduos (18\%) apresentava níveis de pressão arterial compatíveis com hipertensão. Esses indivíduos eram, em sua maioria, mais jovens (menos de 40 anos) do que a amostra total (dados não apresentados). Esse dado merece atenção por se tratar de um grupo possivelmente hipertenso, mas que desconhece a doença, apesar de ser profissional de saúde com amplo conhecimento no assunto e lidar frequentemente com a pressão arterial.

Esse estudo merece atenção por avaliar a dose de exposição ao trabalho noturno em condições do mundo real e usando a pressão arterial aferida. Embora o esfigmomanômetro de mercúrio seja o padrão de excelência para aferição da pressão arterial, os dispositivos automáticos para avaliação da pressão arterial são suficientemente precisos e geram pouco viés nas estimativas do efeito dos fatores de risco relacionados à pressão arterial e à hipertensão. Portanto, tais dispositivos automáticos podem ser usados em grandes estudos epidemiológicos ${ }^{14}$. No entanto, os trabalhadores que foram classificados como hipertensos com base em três medidas pontuais de pressão arterial em intervalos de 1 minuto, mas não há informações mais detalhadas quanto ao horário exato em que a aferição 
da pressão arterial foi realizada. Como a pesquisa de campo foi realizada no horário de trabalho, a pressão arterial dos atuais trabalhadores noturnos foi medida entre $21 \mathrm{~h}$ e $23 \mathrm{~h}$; e de ex-trabalhadores noturnos, entre $9 \mathrm{~h}$ e $18 \mathrm{~h}$. O momento da avaliação é um potencial fator de confusão, uma vez que uma variação circadiana é esperada para a pressão $\operatorname{arterial}^{32}$. Porém, mesmo diante desta limitação, foi possível captar as associações. Certamente, o ideal seria ajustar as análises pelo horário da aferição, caso esses dados estivessem disponíveis.

Um ponto forte desse estudo é a investigação da exposição prévia ao trabalho noturno. No entanto, não havia informações sobre o tempo decorrido desde o término do trabalho noturno até o momento da coleta de dados, o que pode ter gerado heterogeneidade no grupo. Apesar disso, foi possível observar os efeitos adversos da duração do trabalho noturno, que foram mais fortes entre os atuais trabalhadores noturnos do que entre os ex-trabalhadores noturnos (dados não apresentados). O delineamento do estudo contribui para a literatura sobre doses de exposição ao trabalho noturno e aumento da pressão arterial, relação que ainda não foi totalmente esclarecida. Apesar da abordagem transversal, em que a temporalidade dos eventos não pode ser assegurada, é pouco provável que a hipertensão leve os trabalhadores a altas doses de trabalho noturno, o que reforça o potencial deste estudo. Tendo em vista que a amostra era composta em sua maioria por mulheres, seria relevante ajustar as análises para o estado da menopausa, mas infelizmente esse fator não foi medido, o que deve ser levado em consideração na interpretação dos resultados. Participaram do estudo profissionais de enfermagem que trabalhavam 12 horas consecutivas, com folga de 60 horas, o que permite mais de um vínculo empregatício, prática comum nas equipes de enfermagem ${ }^{13}$. Consequentemente, os resultados devem ser generalizados para outras modalidades de trabalho com cautela.

\section{Conclusão}

A abordagem detalhada da exposição ao trabalho noturno, usando a ótica mais refinada de duração e frequência, incorporou novas dimensões às análises, contribuindo assim com maior robustez para a literatura. É possível que aspectos da dose de exposição ao trabalho noturno possam contribuir para controvérsias identificadas na literatura. Por ser um estudo transversal, os resultados apontam para relações causais, mas sugere a possibilidade de que os efeitos do trabalho noturno comecem após certas doses de exposição (mais de 4 noites por quinzena ou mais de 9 anos). Embora a hipertensão tenha origem multifatorial, esse estudo trouxe uma nova perspectiva para a compreensão da hipertensão na saúde do trabalhador. Nesse sentido, os resultados podem contribuir para estratégias que visem minimizar o número de noites trabalhadas pelas equipes de enfermagem do hospital, a fim de reduzir os prejuízos causados pelo trabalho noturno.

Em síntese, os resultados evidenciam a necessidade de atenção aos trabalhadores noturnos, que enfrentam os prejuízos biológicos e psicossociais associados aos esquemas de trabalho. Estratégias eficazes de melhoria da saúde para esses trabalhadores devem ser expandidas, levando em consideração critérios cronobiológicos nas intervenções, a fim de promover a saúde e o bem-estar dos trabalhadores, bem como atendimento eficaz ao paciente.

\section{Contribuicoes de autoria}

Silva-Costa A, Braz BP, Griep RH e Rotenberg L contribuíram substancialmente para o delineamento do estudo, coleta e análise de dados, redação do manuscrito, revisões e aprovação da versão final, e assumem total responsabilidade pelo estudo e pelo conteúdo publicado.

\section{Referências}

1. Instituto Brasileiro de Geografia e Estatística. Pesquisa Nacional por Amostra de Domicílios Contínua 2012/2017 [Internet]. Rio de Janeiro; 2017 [citado em 20 jul 2020]. Disponível em: https://biblioteca.ibge.gov.br/visualizacao/livros/ liv101622_informativo.pdf

2. Brum MCB, Dantas Filho FF, Schnorr CC, Bottega GB, Rodrigues TC. Shift work and its association with metabolic disorders. Diabetol Metab Syndr. 2015;7:45.
3. Anothaisintawee T, Reutrakul S, Van Cauter E, Thakkinstian A. Sleep disturbances compared to traditional risk factors for diabetes development: Systematic review and meta-analysis. Sleep Med Rev. 2016;30:11-24.

4. Manohara S, Thongprayoonb C, Cheungpasitporna W, Maoa MM, Herrmanna SM. Associations of rotational shift work and night shift status with hypertension: a systematic review and meta-analysis. J Hypertens. 2017;35:1929-37. 
5. Sun M, Feng W, Wang F, Li P, Li Z, Li M, et al. Meta-analysis on shift work and risks of specific obesity types. Obes Rev. 2018;19:28-40.

6. Lobo LAC, Canuto R, Costa LSD, Pattussi MP. Tendência temporal da prevalência de hipertensão arterial sistêmica no Brasil. Cad Saude Publica. 2017;33:e00035316.

7. Moreno CRC, Marqueze EC, Sargent C, Wright KP Jr, Ferguson SA, Tucker P. Working Time Society consensus statements: Evidence-based effects of shift work on physical and mental health. Ind Health. 2019;57(2):139-57.

8. Souza BB, Monteze NM, Oliveira FL, Oliveira JM, Freitas NS, Marques NNR, et al. Lifetime shift work exposure: association with anthropometry, body composition, blood pressure, glucose and heart rate variability. Occup Environ Med. 2015;72:208-15.

9. Ferguson JM, Costello S, Neophytou AM, Balmes JR, Bradshaw PT, Cullen MR, et al. Night and rotational work exposure within the last 12 months and risk of incident hypertension. Scand J Work Env Hea. 2019;45:256-66.

10. Rotenberg L, Silva-Costa A, Vasconcellos-Silva P, Griep RH. Work Schedule and self-reported hypertension - the potencial beneficial role of on-shift naps for night workers. Chronobiol Int. 2016;33:697-705.

11. Tucker P, Härmä M, Ojajärvi A, Kivimäki M, Leinbeweber C, Oksanen T, et al. Associations between shift work and use of prescribed medications for the treatment of hypertension, diabetes, and dyslipidemia: a prospective cohort study. Scand J Work Env Health. 2019;8:3813.

12. Rotenberg L, Silva-Costa A, Diniz TB, Griep RH. Long-term deleterious effects of night work on sleep. Sleep Sci. 2011;4:13-20.

13. Fernandes JDC, Portela LF, Griep RH, Rotenberg L. Working hours and health in nurses of public hospitals according to gender. Rev Saude Publica. 2017;51:63.

14. Vera-Cala LM, Orostegui M, Valencia-Angel LI, López N, Bautista LE Accuracy of the Omron HEM-705 CP for blood pressure measurement in large epidemiologic studies. Arq Bras Cardiol. 2011;96(5):393-8.

15. Wang XS, Travis RC, Reeves G, Green J, Allen NE, Key TJ, et al. Characteristics of the Million Women Study participants who have and have not worked at night. Scand J Work Env Health. 2012;38:590-9.

16. Guo Y, Liu Y, Huang X, Rong Y, He M, Wang Y, et al. The effects of shift work on sleeping quality, hypertension and diabetes in retired workers. PLoS One. 2013;8:e71107.

17. Vetter C, Devore EE, Wegrzyn LR, Massa J, Speizer FE, Kawachi I, Rosner B, Stampfer MJ, Schernhammer ES. Association Between Rotating Night Shift Work and Risk of Coronary Heart Disease Among Women. JAMA. 2016;315(16):1726-34.

18. Peplonska B, Bukowska A, Sobala W. Association of Rotating Night Shift Work with BMI and
Abdominal Obesity among Nurses and Midwives. PloS one. 2015;10:e0133761.

19. Ramin C, Devore E, Wang W, Pierre-Paul J, Wegrzyn LR, Schernhammer ES. Night shift work at specific age ranges and chronic disease risk factors. Occup Environ Med. 2015;72:100-7.

20. Park S, Nam J, Lee JK, Oh SS, Kang HT, Koh SB. Association between night work and cardiovascular diseases: analysis of the $3^{\text {rd }}$ Korean working conditions survey. Ann Occup Environ Med. 2015;27:15

21. Silva-Costa A, Rotenberg L, Nobre AA, Schmidt MI, Chor D, Griep RH. Gender-specific association between night-work exposure and type-2 diabetes: results from longitudinal study of adult health, ELSA-Brasil. Scand J Work Env Health. 2015;41:569-78.

22. Puttonnen S, Härmä M, Hublin C. Shift work and cardiovascular disease-pathways from circadian stress to morbidity. Scand J Work Env Health. 2010;36:96-108.

23. Hermida RC. Ambulatory blood pressure monitoring in the prediction of cardiovascular events and effects of chronotherapy: rationale and design of the MAPEC study. Chronobiol Int. 2010;24:749-75.

24. Riegel B, Marguerite L, Lozano A, Malone SK, Patterson F, Hanlon AL. Shift Workers Have Higher Blood Pressure Medicine Use, But Only When They Are Short Sleepers: A Longitudinal UK Biobank Study. J Am Heart Assoc. 2019;8(20):e013269.

25. Birkenhäger AM, Van den Meiracker AH. Causes and consequences of a non-dipping blood pressure profile. Neth J Med. 2007;65:127-31.

26. Kecklund G, Axelsson J. Health consequences of shift work and insufficient sleep. BMJ. 2016;355:i5210.

27. Kim MJ, Lee JH, Duffy JF. Circadian Rhythm Sleep Disorders. J Clin Outcomes Manag. 2016;20:513-28.

28. Arendt J. Shift work: coping with the biological clock. Occup Med. 2010;60:10-20.

29. Pepłońska B, Nowak P, Trafalska E. The association between night shift work and nutrition patterns among nurses: a literature review. Med Pr. 2019;70(3):363-76.

30. Peplonska B, Bukowska A, Wieczorek E, Przybek M, Zienolddiny S, Reszka E. Rotating night work, lifestyle factors, obesity and promoter methylation in BRCA1 and BRCA2 genes among nurses and midwives. PloS one. 2017;2:e0178792.

31. Park J, Shin SY, Kang Y, Rhie J. Effect of night shift work on the control of hypertension and diabetes in workers taking medication. Ann Occup Environ Med. 2019;31:e27.

32. Smolensky MH, Hermida RC, Portaluppi F. Circadian mechanisms of 24-hour blood pressure regulation and patterning. Sleep Med Rev. 2017;33:4-16. 